Review

\title{
Emerging roles of the SUMO pathway in mitosis
} Mary Dasso

\author{
Address: Laboratory of Gene Regulation and Development, NICHD/NIH, Building 18, Room 106, MSC-5431, Bethesda, MD 20892, USA \\ Email: Mary Dasso - mdasso@helix.nih.gov
}

Published: 24 January 2008

Cell Division 2008, 3:5 doi:10.1186/1747-1028-3-5

This article is available from: http://www.celldiv.com/content/3/l/5

(c) 2008 Dasso; licensee BioMed Central Ltd.

This is an Open Access article distributed under the terms of the Creative Commons Attribution License (http://creativecommons.org/licenses/by/2.0), which permits unrestricted use, distribution, and reproduction in any medium, provided the original work is properly cited.
Received: 7 January 2008

Accepted: 24 January 2008

\begin{abstract}
SUMO proteins are small ubiquitin-like modifiers found in all eukaryotes that become covalently conjugated to other cellular proteins. The SUMO conjugation pathway is biochemically similar to ubiquitin conjugation, although the enzymes within the pathway act exclusively on SUMO proteins. This post-translational modification controls many processes. Here, I will focus on evidence that SUMOylation plays a critical role(s) in mitosis: Early studies showed a genetic requirement for SUMO pathway components in the process of cell division, while later findings implicated SUMOylation in the control of mitotic chromosome structure, cell cycle progression, kinetochore function and cytokinesis. Recent insights into the targets of SUMOylation are likely to be extremely helpful in understanding each of these aspects. Finally, growing evidence suggests that SUMOylation is a downstream target of regulation through Ran, a small GTPase with important functions in both interphase nuclear trafficking and mitotic spindle assembly.
\end{abstract}

\section{Introduction}

SUMO proteins are small ubiquitin-like modifiers that become covalently conjugated to cellular proteins. In budding yeast, proteomic experiments indicate that 300 or more proteins may be SUMOylation targets [1-4]. This post-translational modification controls multiple events, including transcription, DNA repair, DNA recombination and mitotic chromosome segregation. The three former processes were covered within recent reviews [5-11], and will not be discussed here. Rather, I will focus on evidence that SUMOylation plays a critical role in mitotic chromosome structure and segregation, and on how this pathway may be regulated during mitosis.

\section{SUMO proteins and their conjugation pathway}

There is one SUMO protein in S. cerevisiae (Smt3p) and $S$. pombe (Pmt3), but mammalian cells typically express three SUMO paralogues (SUMO1-3) [12]. Like ubiquitin, newly translated SUMOs require cleavage to reveal C-terminal diglycine motifs (Figure 1, Step 1). After matura- tion, SUMO1 is $~ 45 \%$ identical to SUMO2 or 3 , while SUMO2 and 3 are $~ 95 \%$ identical to each other. Where they cannot be distinguished, I will refer to SUMO2 and 3 collectively as SUMO2/3. Proteases of the Ubiquitin like protein protease/Sentrin specific proteases (Ulp/SENPs) family catalyze SUMO processing [13]. S. cerevisiae has two Ulp/SENPs (Ulp1p and Ulp2p/Smt4p). Ulp1p associates with the nuclear envelope [14], and is important for Smt3p maturation [15]. S. pombe likewise has two Ulp/ SENPs (also called Ulp1 and Ulp2), while mammals have six (SENP1, 2, 3, 5, 6 and 7) [13].

Conjugation of mature SUMOs occurs through a cascade (Figure 1, Steps 2-4) containing a heterodimeric activating enzyme (E1 enzyme. Uba2/Aos1), a conjugating enzyme (E2 enzyme. Ubc9) and usually a SUMO ligase (E3 enzyme) [12]. Nomenclature of SUMO pathway enzymes in yeast and vertebrates are given in Table 1 . The result of these reactions is an isopeptide linkage between the SUMO C-terminal glycine and an $\varepsilon$-amino group of a 


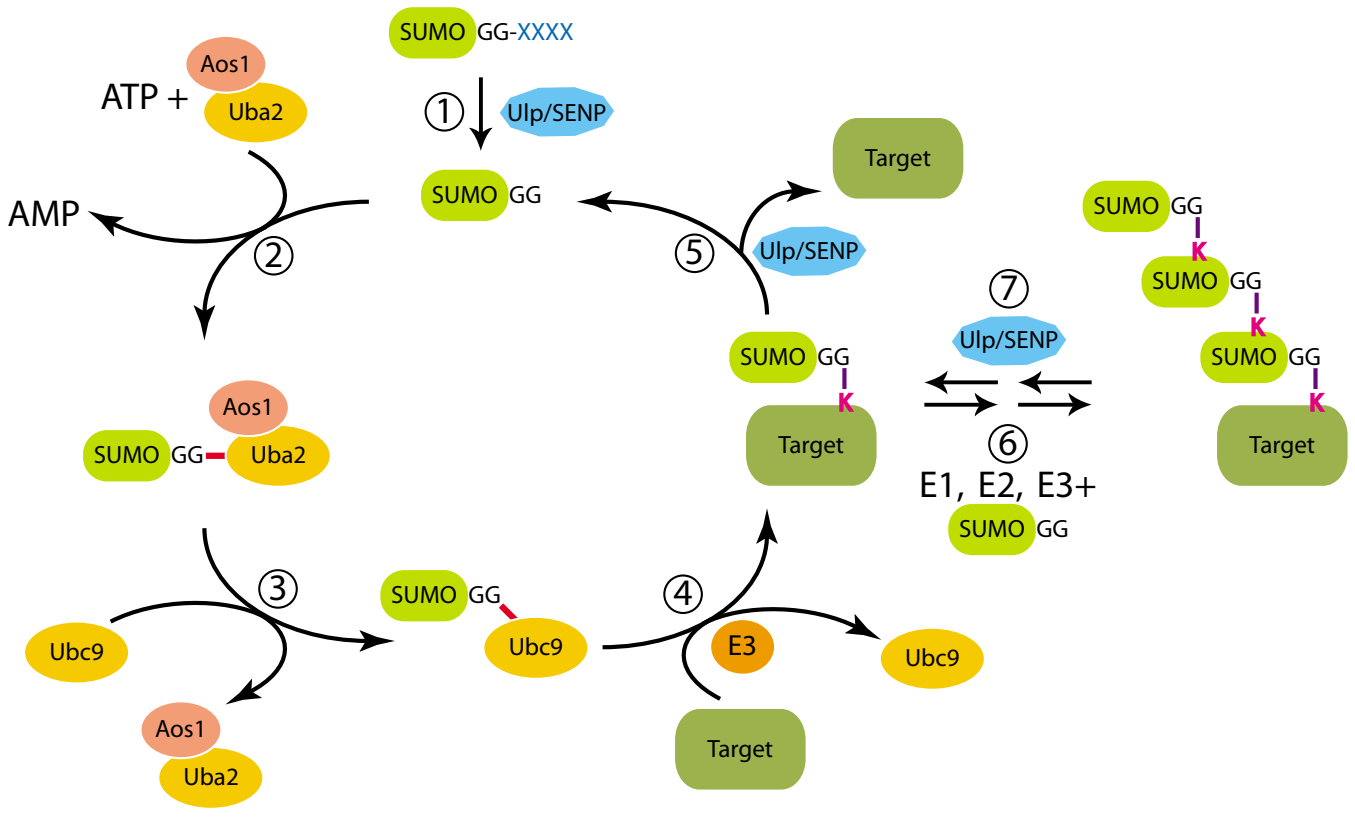

\section{Figure I}

SUMO pathway. SUMO proteins undergo post-translational maturation, catalyzed by Ulp/SENPs, to reveal a C-terminal diglycine motif (Step I). Mature SUMOs undergo ATP-dependent activation, resulting in a thiolester linkage between the C-terminal di-glycine and their activating enzyme, Uba2/Aos I (Step 2). The thiolester is transferred to their conjugating enzyme, Ubc9 (Step 3). Ubc9 acts in concert with SUMO ligases/E3 enzymes to form an isopeptide linkage between the SUMO C-terminus and an $\varepsilon$-amino group of a lysine within the target protein (Step 4). SUMOs can be removed from conjugated species by the action of Ulp/SENPs (Step 5). In some cases, SUMO chains can be formed through linkage of additional SUMO moieties to previously conjugated SUMOs (Step 6). While it is possible that multiple Ulp/SENPs may disassemble SUMO chains (Step 7), members of the Ulp2 family appear to be specialized for this reaction.

lysine within the target protein. The biochemistry of SUMO and ubiquitin conjugation are similar, but no enzymes act on both SUMOs and ubiquitin. On the other hand, the same E1 and E2 enzymes act for the conjugation of all mammalian SUMO paralogues. SUMO-conjugated species are deconjugated by Ulp/SENPs (Figure 1, Step 5), rendering this modification highly dynamic. Smt3p, SUMO2 and SUMO3 can form chains, primarily through a conserved acceptor lysine [16-18] (Figure 1, Step 6). SUMO1 can form chains in vitro through other lysine residues [19], although SUMO1 chains have not been demonstrated in vivo. A subset of Ulp/SENPs is specialized for dismantling of SUMO chains (Figure 1, Step 7): In budding yeast, Ulp2p is predominantly nuclear [20]. Many phenotypes of $u l p 2$ mutants arise from their inability to disassemble Smt3p chains, suggesting that it is critical for this reaction [17]. A related protein, SENP6, has been implicated in chain editing in mammalian cells [21].

There are multiple SUMO E3 enzymes, and it is likely that their specificity plays a significant role in determining the spectrum of SUMOylated species. A conserved group of SUMO E3 enzymes found in all eukaryotes possess variant
RING-finger like domains (SP-RINGs). This class of E3 enzymes are called Siz ( $\underline{\mathrm{S} A P}$ and miz-finger domain) proteins in yeast and PIAS (protein inhibitor of activated STAT) proteins in vertebrates (reviewed in [22]). Budding yeast Siz1p and Siz2p proteins are required for the bulk of Smt3p conjugation $[23,24]$. The SUMO ligase activity of other SP-RING proteins, Mms21p and Zip3p, are required for DNA repair [25-27] and meiotic synaptonemal complex assembly [28], respectively. Vertebrates express five PIAS proteins (PIAS1, PIAS3, PIASx $\alpha$, PIASx $\beta$ and PIASy), which have been implicated in a broad variety processes, including signal transduction, gene expression and genome maintenance. [29]. Humans also express a homologue of Mms21p [30] and two additional SP-RING proteins, hZIMP7 and hZIMP10 [29].

There are other E3 enzymes in vertebrates that have no obvious homologues in yeast. Vertebrate-specific E3s include Pc2, a polycomb group protein [31], and RanBP2, a large nuclear pore protein that localizes to the cytoplasmic face of the nuclear pore complex (NPC) [32]. No mitotic role of Pc2 has been established. However, the interactions and mitotic behavior of RanBP2 has been 
Table I: SUMO Pathway Enzymes

\begin{tabular}{|c|c|c|c|}
\hline Enzymatic activity & Vertebrate & S. cerevisiae & S. pombe \\
\hline \multirow[t]{3}{*}{ SUMO paralogues } & SUMOI & Smt3p & Pmt3p \\
\hline & SUMO2 & & \\
\hline & SUMO3 & & \\
\hline \multirow[t]{2}{*}{ SUMO protease } & SENPI-3, SENP5-7 & UlpIp & UlpI \\
\hline & & Ulp2p/Smt4p & Ulp2 \\
\hline Activating Enzyme (EI) & Uba2/SAE2 +Aos I/SAEI & Uba2p+AosIp & Uba2/Fub2 + Rad31 \\
\hline Conjugating Enzyme (E2) & Ubc9 & Ubc9p & Hus5p \\
\hline \multirow[t]{9}{*}{ SP-RING SUMO ligases (E3) } & PIASI & Sizlp & Plil \\
\hline & PIAS3 & Siz2p/Nfilp, & \\
\hline & PIAS $\times \alpha$ & & \\
\hline & PIASx $\beta$ & & \\
\hline & PIASy & & \\
\hline & Mms2I & Mms2Ip & Nse2p \\
\hline & & Zip3p & \\
\hline & Zimp7* & & \\
\hline & Zimplo* & & \\
\hline \multirow[t]{2}{*}{ Other SUMO ligases (E3) } & RanBP2 & (None known) & (None known) \\
\hline & Pc2 & & \\
\hline
\end{tabular}

*Related to PIAS/Siz family, but SUMO ligase activity not been demonstrated in vitro.

intensively studied. RanBP2 possesses a domain called the IR domain, which is comprised of two short, tandemly repeated sequences (around 50 residues), separated by a 24-residue spacer. IR-containing fragments of RanBP2 have SUMO ligase activity in vitro [32]. This domain is also the site of assembly for a complex that contains Ubc9 and the SUMO1-modified form of RanGAP1 (RanGAP1•SUMO1) [33], which will be called the RRSU complex. RanGAP1 is the activating protein for the abundant small GTPase Ran [34]. Ran is required for many cellular functions, including nucleocytoplasmic trafficking, spindle assembly and cell cycle control. RanBP2 possesses four Ran-binding domains that enhance RanGAP1-mediated GTP hydrolysis by Ran.

\section{Genetic links of SUMOylation and mitosis}

The link between SUMOylation and mitosis was established very early in the history of this field. Even before the discovery of SUMO proteins themselves, it was known that budding yeast Ubc9p is essential for degradation of Btype Cyclins [35], key mitotic regulators that are destroyed through ubiquitination at anaphase onset. Moreover, SMT3 was isolated in yeast screens for temperature-sensitive mutants defective in chromosome segregation [36], and for suppressors of mutations of mif2, a homologue of the vertebrate centromeric CENP-C protein [37]. Ongoing characterization of mutants lacking SUMO pathway components has confirmed that many of them have important roles in mitosis.

Smt3p, Uba2p, Aos1p and Ubc9p are encoded by essential genes in budding yeast, consistent with the central role of SUMO pathway in many aspects of cell physiology [38-
40]. $\Delta$ smt 3 strains of $S$. cerevisiae arrest in early mitosis as large budded cells with short spindles [38]. Progression through mitosis requires the anaphase promoting complex/cyclosome (APC/C), a ubiquitin ligase that is responsible for controlled degradation of B-type Cyclins and other substrates at anaphase onset. The APC/C is controlled through the spindle assembly checkpoint (SAC), a regulatory pathway that monitors spindle formation and inhibits APC/C until all chromosomes are correctly attached and aligned on the metaphase plate. Mitotic arrest of $\Delta s \mathrm{st} 3$ cells does not reflect anaphase inhibition through the SAC [38]. Rather, it results from their inability to appropriately activate the APC/C after the SAC is turned off. A similar defect in APC/C activation is observed in $\Delta$ ubc9 cells [38]. Finally, temperature-sensitive uba2 mutants (uba2-ts) show pronounced hypersensitivity to microtubule destabilizing drugs and early mitotic arrest with short, frequently misaligned spindles at the restrictive temperature [40].

In fission yeast, the gene encoding Pmt3p is not essential, nor are the genes encoding the homologues of Uba2p or Ubc9p, although mutants lacking any of these proteins show very slow growth. pmt $3 \Delta$ cells show a spectrum of phenotypes, which are indicative of problems in mitotic chromosome structure or segregation errors [41]. These defects include high frequency loss of mini-chromosomes and a cut (cell untimely torn) phenotype. Similar chromosome segregation phenotypes are found in strains with mutations in S. pombe Aos1 [42] and Ubc9 [43] homologues. In a manner that is perhaps related, transgenic mice lacking Ubc9 show aberrant chromosome structures in mitosis, including hypercondensation and breakage, as 
well as a high rates of missegregation and polyploidy [44]. These defects result in early embryonic lethality in Ubc9deficient mouse embryos $(<$ E7.5).

Mutations in yeast genes encoding SUMO ligases do not cause strong mitotic phenotypes. This observation might either suggest that E3 enzymes function redundantly for key substrates, or that the low level of conjugation obtained by Ubc9 alone is sufficient for mitosis. While most Smt3p conjugation is lost in mutants lacking Siz1p and Siz2p, such double mutants remain viable and grow without pronounced defects in the absence of $2 \mu \mathrm{m}$ circles [45]. $2 \mu \mathrm{m}$ circles are stable extrachromosomal elements that are carried in multiple copies by most budding yeast strains, although they are not necessary for viability.

There is more direct evidence of mitotic requirements for individual E3 enzymes in vertebrates: PIASy is required for chromosome segregation in Xenopus egg extracts [46] and mammalian tissue culture cells [47]. Surprisingly, transgenic mice lacking PIASy are viable, morphologically normal and fertile [48]; this finding suggests that other PIAS proteins may complement SUMOylation deficiencies in PIASy $/$ - mice, or that cells are able to bypass this requirement in vivo through other mechanisms. Separately, numerous observations indicate that the vertebrate RanBP2 protein has an important mitotic role, particularly in promoting the correct attachments of kinetochore microtubules (kMTs) to kinetochores [49-52]. However, it has not been demonstrated that the capacity of RanBP2 to promote such attachments requires its activity as a SUMO ligase.

Finally, SUMO proteases are important within mitosis. Budding yeast ulp1 mutants show cell cycle delays at the $\mathrm{G}_{2} / \mathrm{M}$ boundary and elevated chromosome mis-segregation [15], and these defects are exacerbated by the presence of $2 \mu \mathrm{m}$ circles [53]. Budding yeast Ulp2p is not required for vegetative growth, although it is important for meiosis, chromosome segregation and recovery from DNA replication and spindle assembly checkpoint arrests $[20,54]$. During mitosis, ulp2 mutants show precocious loss of centromeric cohesion [55] and defects in rDNA condensation [20]. Like its budding yeast counterpart, $S$. pombe Ulp1p localizes to the nuclear envelope [56], and ulp1s strains show pronounced growth defects, resembling mutants deficient in the fission yeast Aos1p or Ubc9p homologues. Moreover, fission yeast lacking Ulp1p develop elongated and irregular morphologies in comparison to wild-type cells, with frequent mis-localization of the nucleus within the cell and increased incidence of multiple septations [56].

\section{Mitotic targets of SUMOylation}

Mitotic targets for SUMOylation have been documented from yeast to vertebrates. I will use budding yeast nomenclature for particular targets, unless explicitly stated otherwise. Both vertebrate and budding yeast names are provided in Figure 2 for substrates whose mitotic conjugation has been confirmed. In many cases, the role of SUMOylation in regulating individual targets is poorly understood, frequently not extending beyond their initial identification in broad proteomic screens [1-4]. Despite these problems, it is worth discussing target proteins because their variety suggests that SUMOylation is

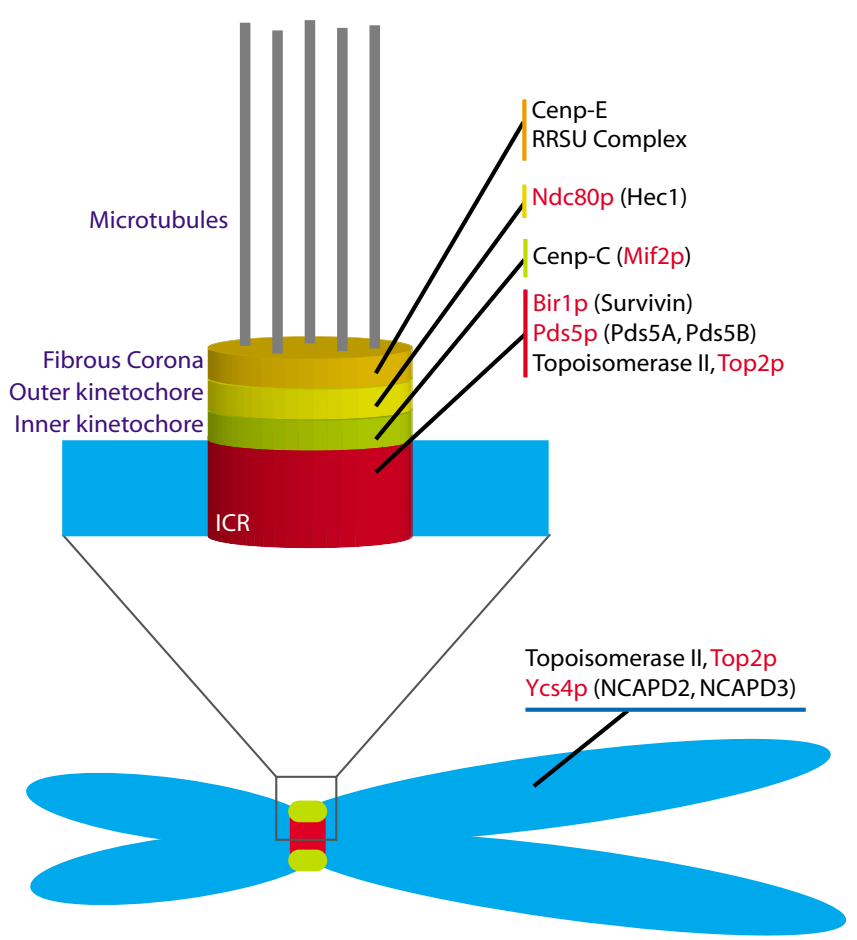

\section{Figure 2}

Mitotic chromosomal SUMOylation substrates. The distribution of SUMOylation substrates is schematically represented, based on the localization of the vertebrate homologues. (The localization reflects the bulk of each protein on mitotic vertebrate chromosomes, not specifically the SUMOylated forms.) The names of vertebrate proteins are indicated in black, while corresponding budding yeast proteins are given in red. In cases where SUMOylation has not been confirmed in both vertebrates and yeast, parentheses indicate the homologue for which demonstration is lacking. This representation does not include many proteins identified in proteomic screens [1-4] whose conjugation has not been independently verified, nor yeast proteins without obvious vertebrate homologues (e.g., Ndc I0p, Cep3p [63]). Confirmed SUMOylation substrates associated to mitotic chromosomes (e.g., Histones [95]) are similarly not represented if the timing of their modification has not been demonstrated within the cell cycle. 
required for numerous aspects of mitosis. This review will consider three classes mitotic targets: namely, proteins with a general role in mitotic chromosome structure, proteins with specific roles at the kinetochore and cytosolic SUMOylation targets.

\section{Mitotic Chromosome Structure: Condensin, Cohesin and Topoisomerase II}

The Condensin and Cohesin complexes are among the most intriguing mitotic SUMOylation targets. Both com-

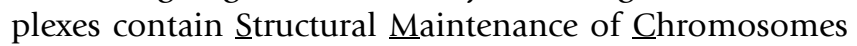
(SMC) proteins. SMCs are large proteins that form rodshaped structures through antiparallel coiled-coil interactions, with an ATP-binding globular domain at one end and a hinge domain at the other [57]. Eukaryotes have six SMC family members, which form heterodimers in specific combinations: Smc1p and Smc3p are constituents of Cohesin complexes, which maintains sister chromatid cohesion until the onset of anaphase. Smc2p and Smc4p constitute the core of Condensin complexes, which maintain the condensed structure of mitotic chromosomes. SMC proteins within both of these complexes (Smc1p, Smc3p, Smc4p) have been identified as SUMOylation substrates in budding yeast through proteomic screens $[1,4]$.

Smc1p and Smc3p associate with non-SMC subunits Scc1p and Scc3p to form Cohesin [57]. Like Smc1p and Smc3p, Scc1p was identified in proteomic searches for yeast SUMOylation targets $[1,4]$. In vertebrates, much of the Cohesin is released from chromosome arms during prophase through the action of the Plk1 and Aurora B kinases. The remaining vertebrate Cohesion is concentrated in the pericentromeric region. In both vertebrates and yeast, all residual Cohesin is released at anaphase through cleavage of the Scc1p by a specific protease, called Separase [57]. Before anaphase, Separase is inhibited through interaction with Securin, a key APC/C target, whose degradation allows Separase activity. The Pds5p protein associates with Cohesin and modulates its binding to chromosomes. Yeast with temperature sensitive mutations in PDS5 ( $p d s 5-1)$ can establish but cannot maintain cohesion, and undergo precocious sister chromatid separation at the restrictive temperature [58]. Pds5p is SUMOylated in a cell cycle-dependent manner, beginning in S-phase and peaking immediately prior to anaphase onset [58]. ULP2 acts as a high-copy suppressor of the cohesion defect in $p d s 5-1$ and other strains with $t s$ alleles of PDS5, but not $p d s 5$ null alleles. Conversely, overexpression of Siz1p significantly enhances the sensitivity of ts pds5 strains. Consistent with these results, increased Ulp2p levels decrease Pds5p SUMOylation, while elevated Siz1p enhances Pds5p modification. These findings suggest that Pds5p interacts with the Cohesin complex to increase the strength of sister chromatid cohesion, and that SUMOylation disrupts this interaction to facilitate cohesion release. It is not yet clear how Pds5p SUMOylation works in parallel with Scc1p proteolysis by Separase, nor how SUMOylation of other Cohesin subunits controls Cohesin function.

Within the Condensin complex, Smc2p and Smc4p associate with non-SMC subunits Ycs4p, Ycs5p and Brn1p. A genetic relationship between Smt3p and Condensin was originally suggested by the finding that Ulp2p overexpression suppresses the smc2-6 allele, and from the observation that $\Delta$ ulp2 yeast are deficient in targeting of the Condensin complex to mitotic chromatin, particularly to rDNA [20]. In addition to Smc4p, Ycs4p and Brn1p were identified as potential SUMOylation targets in yeast $[1,4]$. Functional ramifications of SUMOylation have not been demonstrated for individual Condensin subunits, with the exception of Ycs4p. A fraction of Ycs4p becomes SuMOylated immediately prior to mitosis, with more highly SUMOylated forms peaking during anaphase [59]. The SUMOylation of Ycs4p during anaphase depends upon Cdc14p, a phosphatase that promotes Condensin association with rDNA. These findings might suggest that Cdc14p promotes SUMOylation of Ycs4p, which in turn facilitates Condensin localization to the rDNA during anaphase. On the other hand, inactivation of Ulp2p results in reduced of Ycs4p and other Condensin subunits with the nucleolus and inefficient rDNA segregation $[20,59]$, arguing against a simple antagonistic relationship between these two enzymes in Ycs4p targeting, and suggesting that this pathway has considerable complexity.

Finally, Topoisomerase II is a major target of SUMOylation in both budding yeast and vertebrates. In yeast, ulp $2 \Delta$ strains show precocious loss of centromeric cohesion in a manner that is not dependent upon Scc1p cleavage or redistribution of the Cohesin complex [55]. This loss is suppressed by overexpression of Top $2 \mathrm{p}$, the yeast Topoisomerase II homologue. Top2p is itself SUMOylated, and top2 mutants that lack SUMO conjugation sites are efficient suppressors of the cohesion defect in ulp $2 \Delta$ strains. These findings collectively suggest that Top $2 \mathrm{p}$ plays an important role in centromeric cohesion, which is downregulated through its SUMOylation [55]. In mitotic Xenopus egg extracts, Topoisomerase II is a major substrate for PIASy- and chromatin-dependent conjugation with SUMO2/3 $[46,60]$. A small fraction of the Topoisomerase II assembled onto mitotic chromosomes in Xenopus egg extract is resistant to high salt extraction. Inhibition of SUMO modification by a dominant-negative Ubc9 mutant (dnUbc9) dramatically increases the amount of unmodified Topoisomerase II retained within this population. At the same time, egg extracts treated with dnUbc9 fail to segregate their chromosomes in anaphase, consistent with the possibility that SUMO-dependent re-mode- 
ling of Topoisomerase II promotes release of the tightly bound population and of cohesion.

\section{Centromeres and kinetochores}

Centromeres are epigenetically-specified chromatin domains on each chromosome that facilitate the accurate segregation sister chromatids during mitosis [61]. Kinetochores are proteinaceous structures that assemble on the centromere of each mitotic sister chromatid. They serve as sites of spindle microtubule (MT) attachment. The kinetochore fibers (k-fibers) that link mammalian kinetochores to spindle poles contain both MTs that are directly attached to the kinetochores at their plus ends (kMTs) and MTs that are not [62]. Kinetochore-kMT attachment is monitored through the SAC [61]. The inner centromeric region (ICR) lies between sister centromeres, and contains proteins required for the regulation of sister chromatid cohesion and kMT attachment. Multiple functions of SUMOylation have been proposed within mitotic centromeres and kinetochores, and recent reports have suggested that many proteins within these domains are SUMOylation targets.

In mitotic Xenopus egg extracts, the ICR contains an abundance of SUMO2/3-conjugated species that are modified in a PIASy-dependent manner [46]. Proteomic analysis in budding yeast has identified numerous kinetochore- or centromere-associated SUMOylation targets by mass spectrometry, including: Bir1p [4], Cbf1p [1,4], Cbf5p [4], Mcm21p [4], Ndc10p [4], Ndc80p [1], Sli15p [4] and Slk19p [1]. Montpetit et al. subsequently confirmed and analyzed the SuMOylation of Ndc10p, Bir1p and Ndc80p, as well as SUMOylation of Cep3p [63]. Ndc10p and Cep $3 p$ are subunits of centromeric DNA binding factor 3 (CBF3), a four-protein complex that binds to an essential element within centromeric DNA [64]. Bir1p and Sli15p link CBF3 to MTs in vitro, and may sense tension to activate the Ipl1p kinase in the vicinity of syntelic attachments [65]. The vertebrate homologues of Bir1p, Sli15p and Ipl1p are Survivin, INCENP and the Aurora B kinase, respectively [66]. Survivin, INCENP and Aurora B constitute the chromosomal passenger complex (CPC) in combination with Dasra-B [66]. The CPC is a key player in the spatial and temporal ordering of mitosis, which controls kinetochore-MT interactions, sister chromatid cohesion and cytokinesis. Ndc10p, Cep3p and Bir1p are deSUMOylated by Ulp2p in response to nocodazole, suggesting that their modification is regulated through SAC activation [63]. Mutants that eliminate Ndc10p modification cause chromosome instability, mislocalization of Ndc10p from the mitotic spindle, abnormal anaphase spindles, and a loss of Bir1p SUMOylation, suggesting an important role for SUMO conjugation in yeast kinetochore function [63].
Interestingly, the fission yeast of Aurora kinase Ark1p interacts with two RING-finger proteins that possess $\mathrm{N}$ terminal SUMO interaction motifs (SIMs), called Rfp1p and Rfp $2 p$ [67]. These proteins and their budding yeast homologues recognize SUMOylated proteins, and heterodimerize with Slx8p, another RING-finger protein, to form a functional ubiquitin ligase [67-69]. The demonstration of this ubiquitin ligase activity has suggested a novel paradigm for SUMO-directed destruction of proteins through the ubiquitin-proteasome system. Given the physical association of Ark1p with Rfp1p and Rfp2p, it is attractive to speculate that CPC SUMOylation may direct the ubiquitination of ICR proteins, possibly including $\mathrm{CPC}$ members themselves.

Centromere associated protein C (CENP-C) is a vertebrate homologue of the budding yeast Mif2p protein [37]. CENP-C binds to alpha satellite DNA within centromeres [70]; it is required for the correct assembly of inner kinetochores, as well as checkpoint signaling and accurate chromosome segregation [71]. A temperature-sensitive CENP-C mutant cell line (ts4-11) derived from chicken DT40 cells, shows metaphase delay, but eventually proceeds through mitosis with chromosome missegregation and undergoes arrest within $G_{1}$ phase [72]. This cell cycle arrest phenotype was utilized to screen for human cDNAs that could rescue ts4-11 cells, and SUMO1 was identified within this screen. This finding was interesting in light of the genetic relationship between MIF2 and SMT3 in budding yeast [37], as it suggest that SUMOylation regulates CENP-C in a manner that is conserved between vertebrates and fungi. While CENP-C can be SUMOylated under in vitro conditions [73], it should be noted that the capacity of SUMO1 to rescue ts4-11 cells may equally reflect the modification of other inner kinetochore proteins that interact with CENP-C.

In the outer kinetochore, both $\mathrm{Ndc} 80 \mathrm{p}$ and the vertebrate centromere associated protein E (CENP-E) have been strongly implicated as SUMOylation targets. The Ndc80 complex is a conserved set of kinetochore proteins that consists of Ndc80p, Nuf2p, Spc24p, and Spc25p [74,75]. This complex is essential for metaphase chromosome alignment and anaphase chromosome segregation. Ndc80p is SUMOylated throughout the cell cycle, but this modification is not responsive to the SAC and its functional significance is not clear [63]. SUMOylation of Ndc80p's vertebrate homologue, Hec1, has not yet been reported. CENP-E is a plus end-directed microtubule motor of the kinesin superfamily that localizes to the outer plate of the kinetochore and fibrous corona $[76,77]$. It is important for the congression of chromosomes with single unattached kinetochores to the metaphase plate [78], for the maintenance of bipolar attachment of microtubules to kinetochores, for generation of tension across 
sister kinetochores and for the SAC $[79,80]$. Matunis and colleagues have recently shown that suppression of mitotic SUMOylation in HeLa cells by overexpression of SENP2 leads to a chromosome segregation defect through disruption of CENP-E targeting to kinetochores [81]. They further observed CENP-E itself is both a SUMO2/3 substrate and polySUMO2/3 binding protein. The latter activity was particularly critical, since mutation of a SUMO2/3 interacting motif (SIM-2/3) blocked kinetochore recruitment of CENP-E.

Finally, as discussed above, vertebrate RanGAP1 •SUMO1 and Ubc9 bind RanBP2 [82,83], forming a RanBP2/ RanGAP1•SUMO1/Ubc9 complex (RRSU complex). The SUMOylation of RanGAP1 is indispensable for assembly of the RRSU complex [82], which remains stable throughout the cell cycle [50]. The RRSU complex is targeted to outer kinetochores or fibrous corona in a MT-dependent fashion [50], and plays an important role in k-fiber assembly [49]. Under conditions where RRSU complex targeting is disrupted, kinetochores fail to maintain discrete end-on attachments to single k-fibers and showed a resultant elevation in chromosome mis-segregation [49]. Interestingly, there is substantial variability between vertebrate species and cell types in the amounts of RRSU recruited to kinetochores, with commensurate variability in the extent of chromosome mis-segregation caused by its displacement. [84]. The assembly of the RRSU complex appears to be is restricted to vertebrates, since the IR domain is not found in RanBP2 homologues within flies, worms or fungi [34]. In some other species, particularly plants, RanGAP1 is be targeted to spindles and kinetochores through mechanisms that are not dependent upon SUMOylation [85].

Non-chromosomal mitotic SUMOylation targets: Septins and hNinein Septins are conserved GTP-binding proteins that link cellular membranes with the MT and actin cytoskeletons [86]. They polymerize to $10-\mathrm{nm}$ filaments that serve as organizational scaffolds and that restrict diffusion between different membrane domains. Johnson and Blobel [87] identified Septins Cdc3p, Cdc11p and Shs1p as targets that give rise to the most abundant Smt3p-conjugated species during mitosis in budding yeast. This modification is highly controlled in both time and space: Conjugated Septins appear just before anaphase onset and disappear abruptly at cytokinesis, and only Septins on the mother cell side of the bud neck become modified. It is interesting to speculate that this asymmetry may be related to the polarized distribution of kinases and other cell cycle regulators involved in the budding yeast morphogenesis checkpoint, which triggers cell cycle arrest in response to insults affecting the actin or septin cytoskeleton [88]. As discussed below, the Septin SUMOylation pattern reflects tightly regulated re-localization of both conjugation and deconjugation enzymes [89]. While SUMOylation of metazoan Septins has not been reported, it has been demonstrated that Drosophila Septins can interact with components of the SUMO conjugation machinery in vitro, and that these components are re-localized during mitosis in a manner that would allow association in vivo [90].

Construction of a SUMOylation site triple mutant that eliminated conjugation of Cdc3p, Cdc11p and Shs1p abolished almost all mitotic SUMOylation at the bud neck, and drastically decreased the overall level of SUMOylation within $\mathrm{G}_{2} / \mathrm{M}$ phase budding yeast cells [87]. This triple mutant was unable to correctly disassemble of Septin filaments, and thus retained persistent Septin rings from previous divisions. This phenotype demonstrates that SUMO conjugation is important for Septin ring dynamics during the cell cycle. Despite these drastic changes in SUMOylation patterns, however, the triple SUMOylation mutant grew without defect, showed no sensitivity to stress conditions, and did not exacerbate the phenotype of a $u b a 2-t s 10$ strain. The two other Septins expressed during vegetative growth, Cdc10p [3] and Cdc12p [1], have subsequently been found as SUMOylation targets within proteomic analysis. It is possible that low levels of mitotic SUMOylation of these Septins may have compensated for the absence of Cdc3p, Cdc11p and Shs1p modification within the triple mutant. This idea might be consistent with the fact that the triple mutant was synthetically lethal at $25^{\circ} \mathrm{C}$ with a $c d c 12-1$ temperature sensitive allele.

Finally, there is some indication that SUMOylation plays a role at centrosomes. hNinein is a human centrosomal protein involved in MT nucleation and anchoring at the centrosome; it binds SUMO1, can be SUMOylated in vitro, and may also be conjugated in vivo [91]. Overexpression of SUMO1 can cause the recruitment of hNinein to foci within interphase nuclei, along with Pericentrin, a protein that anchors regulatory and structural molecules to centrosomes, and $\gamma$-tubulin, the primary mediator of centrosomal MT nucleation. Pericentrin also interacts with CHD3/ZFH [92]. CHD3/ZFH is a member of the chromodomain-SWI/SNF helicase family, and it is also a SUMObinding protein [93].

\section{Regulation of SUMOylation within mitosis}

Mitotic SUMOylation is likely to be highly regulated, both spatially and temporally. Intriguingly, there are several links of this regulation to the Ran GTPase pathway [34]. As mentioned above, RanGAP1 is cytoplasmic during interphase. Vertebrate RanGAP1 it is targeted to the NPC in a SUMO1-dependent fashion through binding to RanBP2 within the RRSU complex. Ran's sole nucleotide exchange factor, RCC1, binds chromatin throughout the 
cell cycle. The distribution of Ran's regulators leads to relatively high concentrations of Ran-GTP in interphase nuclei, and in the vicinity of mitotic chromatin. Local Ran-GTP levels provide cues required for correct spatial and temporal organization of cells. The main effectors of this pathway are a family of Ran-GTP-binding nuclear transport receptors that are collectively called karyopherins. Karyopherins can associate with interphase NPCs and freely traverse them in a Ran-independent manner. They interpret the high relative levels of Ran-GTP within nuclei to direct compartment-specific loading or unloading of cargo: Import receptors (Importins) bind to their cargo in the cytoplasm, traverse the NPC and dissociate upon RanGTP binding. Export receptors (Exportins) bind their cargo inside nuclei in ternary complexes that contain RanGTP. After passage through the NPC, export complexes dissociate upon Ran-GTP hydrolysis.

During mitosis, the Exportin Crm1 directs in RRSU localization to kinetochores $[49,50]$. Crm 1 binds kinetochores in a manner that requires neither Ran-GTP nor MTs. However, inhibition of Crm1 ternary complex formation by leptomycin B (LMB), a highly specific chemical inhibitor, blocks kinetochore recruitment of RRSU in HeLa and U2OS cells [49]. Kinetochores of LMB-treated cells show increased tension, and frequently associate with continuous microtubule bundles that span their centromeres, indicating that their k-fibers do not maintain discrete endon attachments to individual kinetochores. Since the RRSU complex contains both Ubc9 and RanBP2, a highly active E3 enzyme in vitro, it is attractive to speculate that these defects may reflect an inability of these enzymes to appropriately modify kinetochore-bound targets. On the other hand, it is unclear whether the RRSU acts as an E3 enzyme in vivo, since analysis using purified domains of RRSU components has suggested that incorporation of Ubc9 into the complex inhibits its E2 activity [33]. It is possible that the fragments used for in vitro studies may not accurately reflect the interactions of the full-length proteins. A more intriguing idea might be that this inhibition occurs in vivo but is released by some signal(s), thus subjecting SUMOylation by RRSU to biological control.

Karyopherins also regulate budding yeast Ulp1p association to the NPC. NPC localization of Ulp1p is not required for its enzymatic activity, but rather restricts its action on nucleoplasmic substrates and promotes efficient deconjugation of proteins at the NPC [14]. The NPC localization of Ulp1p is maintained through targeting sequences within the N-terminus of Ulp1p that interacts with two Importins, Pse1p and Importin- $\alpha / \beta$ [94]. The binding of Ulp1p to Pse1p or Importin- $\alpha / \beta$ is unusual because Ulp $1_{1-404}$ is not released from either receptor in vitro by Ran-GTP [94], and it has been suggested that the refractory nature of this association may be important for the stability of NPC localization. Despite the stability of such interactions, they must be remodelled at the end of mitosis to allow Ulp1p-mediated deconjugation of cytoplasmic substrates, particularly Septins. It appears that these two karopherins have a complex and perhaps antagonistic roles in this process [89]: Ulp1p mutants that cannot bind Importin- $\alpha / \beta$ target aberrantly to the Septin ring in large budded cells, suggesting that Importin- $\alpha / \beta$ normally antagonizes recruitment of Ulp 1 to the bud neck. Pse1p is required for this Ulp1p recruitment to the Septin Ring, and it appears that the alterations in the association of Pse1p to the NPC may be primarily responsible for allowing Ulp1p to act on Septins.

Finally, conjugation through PIAS family proteins is regulated in mitosis. For Siz1p in budding yeast, this is again accomplished through the activity of Karyopherins [89]. Importin- $\alpha / \beta$ imports Siz1 $p$ into the nucleus during interphase. Siz1p is phosphorylated in mitosis, and this may allow its export from the nucleus by the Exportin Msn5p, followed by recruitment to the Septin ring, where it mediates Septin SUMOylation. In vertebrate systems, nuclear compartmentalization is not likely to play the same role in controlling mitotic SUMOylation because vertebrates undergo open mitosis. In metazoans, it has been demonstrated that PIAS proteins are controlled through their differential recruitment to mitotic chromosomes [46]. Specifically, PIASy concentrates on mitotic chromosomes in Xenopus egg extracts; PIASx $\alpha$ shows a lower level of accumulation, but other PIAS proteins are not recruited to mitotic chromatin. Other PIAS proteins do not recruit Ubc9 onto the chromatin at levels comparable to PIASy, nor do they restore the capacity of PIASy-depleted extracts to modify Topoisomerase-II and other chromatin-bound targets. Mechanisms that may underlie the specificity of PIASy recruitment will obviously be important topics for further investigation.

\section{Summary}

The SUMO pathway has been implicated in several aspects of mitosis, including chromosome structure, cell cycle progression, kinetochore function and cytokinesis. While these findings are clearly intriguing, much remains to be understood. Questions of particular interest will include:

1. A large number of potential SUMOylation targets have been identified through proteomic screens. While it is too soon to predict that all of these proteins are SUMOylated under physiological conditions, it seems likely that the majority of them will be genuine targets. It will be a major task to validate their status as targets, to determine the circumstances under which they are modified, and to find whether such modification is conserved between species. 
2. Even for those substrates that have been well-documented as mitotic SuMOylation targets, we have almost no understanding of how this modification alters their function at a molecular level. It is likely that several paradigms will emerge, including the use of SUMOylation to direct target localization, to alter protein-protein interactions and to select targets for ubiquitin-mediated degradation. Discovering these mechanisms should offer fascinating examples of biological regulation.

3. Finally, it is attractive to speculate that the modification of proteins that mediate a variety of mitotic processes may be coordinated, in order to facilitate the temporal and spatial organization of these processes with respect to each other. As we understand the role of SUMOylation for individual targets, it will therefore be of great interest to examine whether and how these targets are linked to each other. Equally, it will be important to understand how this pathway fits into better defined regulatory schemes featuring mitotic kinases and controlled protein degradation.

\section{Acknowledgements}

This work was entirely supported by NICHD Intramural funds. I would like to thank Yoshiaki Azuma and Alexei Arnaoutov for helpful discussions and comments.

\section{References}

I. Denison C, Rudner AD, Gerber SA, Bakalarski CE, Moazed D, Gygi SP: A proteomic strategy for gaining insights into protein sumoylation in yeast. Mol Cell Proteomics 2005, 4(3):246-254.

2. Hannich JT, Lewis A, Kroetz MB, Li SJ, Heide H, Emili A, Hochstrasser $M$ : Defining the SUMO-modified proteome by multiple approaches in Saccharomyces cerevisiae. J Biol Chem 2005, 280(6):4I02-4II0.

3. Panse VG, Hardeland U, Werner T, Kuster B, Hurt E: A proteomewide approach identifies sumoylated substrate proteins in yeast. J Biol Chem 2004, 279(40):4|346-4I35I.

4. Wohlschlegel JA, Johnson ES, Reed SI, Yates JR 3rd: Global analysis of protein sumoylation in Saccharomyces cerevisiae. I Biol Chem 2004, 279(44):45662-45668.

5. Gill G: Something about SUMO inhibits transcription. Curr Opin Genet Dev 2005, I 5(5):536-54l.

6. Hay RT: SUMO: a history of modification. Mol Cell 2005, I 8(I): I- 12 .

7. Hay RT: Role of ubiquitin-like proteins in transcriptional regulation. Ernst Schering Res Found Workshop 2006:173-192.

8. Moschos S], Mo YY: Role of SUMO/Ubc9 in DNA damage repair and tumorigenesis. I Mol Histol 2006, 37(5-7):309-319.

9. Pastushok L, Xiao W: DNA postreplication repair modulated by ubiquitination and sumoylation. Adv Protein Chem 2004, 69:279-306

10. Seeler JS, Bischof O, Nacerddine K, Dejean A: SUMO, the three Rs and cancer. Curr Top Microbiol Immunol 2007, 3 I 3:49-7I.

II. Ulrich HD: PCNA(SUMO) and Srs2: a model SUMO substrate-effector pair. Biochem Soc Trans 2007, 35(Pt 6): I 385-1 388

12. Johnson ES: Protein modification by SUMO. Annu Rev Biochem 2004, 73:355-382.

13. Mukhopadhyay D, Dasso M: Modification in reverse: the SUMO Proteases. Trends in Biochem Sci 2007, in press:.

14. Li SJ, Hochstrasser M: The Ulp I SUMO isopeptidase: distinct domains required for viability, nuclear envelope localization, and substrate specificity. J Cell Biol 2003, I60(7): |069-108I.

15. Li SJ, Hochstrasser M: A new protease required for cell-cycle progression in yeast. Nature I999, 398(6724):246-25I.
16. Bencsath KP, Podgorski MS, Pagala VR, Slaughter CA, Schulman BA: Identification of a multifunctional binding site on Ubc9p required for Smt3p conjugation. J Biol Chem 2002, 277(49):47938-47945.

17. Bylebyl GR, Belichenko I, Johnson ES: The SUMO isopeptidase Ulp2 prevents accumulation of SUMO chains in yeast. J Biol Chem 2003, 278(45):44II3-44I 20.

18. Tatham MH, Jaffray E, Vaughan OA, Desterro JM, Botting $\mathrm{CH}$, Naismith JH, Hay RT: Polymeric chains of SUMO-2 and SUMO-3 are conjugated to protein substrates by SAEI/SAE2 and Ubc9. J Biol Chem 200I, 276(38):35368-35374.

19. Pedrioli PG, Raught B, Zhang XD, Rogers R, Aitchison J, Matunis M Aebersold R: Automated identification of SUMOylation sites using mass spectrometry and SUMmOn pattern recognition software. Nat Methods 2006, 3(7):533-539.

20. Strunnikov AV, Aravind L, Koonin EV: Saccharomyces cerevisiae SMT4 encodes an evolutionarily conserved protease with a role in chromosome condensation regulation. Genetics 200I, I 58(I):95-107.

21. Mukhopadhyay D, Ayaydin F, Kolli N, Tan SH, Anan T, Kametaka A Azuma $Y$, Wilkinson KD, Dasso M: SUSPI antagonizes formation of highly SUMO2/3-conjugated species. J Cell Biol 2006, I 74(7):939-949.

22. Hochstrasser M: SP-RING for SUMO: new functions bloom for a ubiquitin-like protein. Cell 200I, I 07(I):5-8.

23. Takahashi $Y$, Kahyo T, Toh EA, Yasuda H, Kikuchi $Y$ : Yeast UIII/ Sizl is a novel SUMOI/Smt3 ligase for septin components and functions as an adaptor between conjugating enzyme and substrates. J Biol Chem 200I, 276(52):48973-48977.

24. Johnson ES, Gupta AA: An E3-like factor that promotes SUMO conjugation to the yeast septins. Cell 200I, I 06(6):735-744.

25. Lee KM, O'Connell MJ: A new SUMO ligase in the DNA damage response. DNA Repair (Amst) 2006, 5(I):|38-|4|.

26. Potts $P R, Y u H$ : Human MMS2I/NSE2 is a SUMO ligase required for DNA repair. Mol Cell Biol 2005, 25( I 6):702 I-7032.

27. Zhao X, Blobel G: A SUMO ligase is part of a nuclear multiprotein complex that affects DNA repair and chromosomal organization. Proc Natl Acad Sci U S A 2005, I 02(I 3):4777-4782.

28. Cheng $\mathrm{CH}$, Lo YH, Liang SS, Ti SC, Lin FM, Yeh CH, Huang HY, Wang TF: SUMO modifications control assembly of synaptonemal complex and polycomplex in meiosis of Saccharomyces cerevisiae. Genes Dev 2006, 20( I 5):2067-208I.

29. Sharrocks AD: PIAS proteins and transcriptional regulation-more than just SUMO E3 ligases? Genes Dev 2006, 20(7):754-758.

30. Potts PR, Yu H: The SMC5/6 complex maintains telomere length in ALT cancer cells through SUMOylation of telomere-binding proteins. Nat Struct Mol Biol 2007, I 4(7):58I-590.

31. Kagey $\mathrm{MH}$, Melhuish TA, Wotton D: The polycomb protein Pc2 is a SUMO E3. Cell 2003, II3(I): I27-137.

32. Pichler A, Gast A, Seeler JS, Dejean A, Melchior F: The nucleoporin RanBP2 has SUMOI E3 ligase activity. Cell 2002, I08(I): 109-120.

33. Reverter D, Lima CD: Insights into E3 ligase activity revealed by a SUMO-RanGAPI-Ubc9-Nup358 complex. Nature 2005, 435(7042):687-692

34. Dasso M: The Ran GTPase: theme and variations. Curr Biol 2002, I 2( I 4):R502-8

35. Seufert W, Futcher B, Jentsch S: Role of a ubiquitin-conjugating enzyme in degradation of S- and M-phase cyclins. Nature 1995, 373(6509):78-8I.

36. Biggins S, Bhalla N, Chang A, Smith DL, Murray AW: Genes involved in sister chromatid separation and segregation in the budding yeast Saccharomyces cerevisiae. Genetics 200I, I 59(2):453-470.

37. Meluh PB, Koshland D: Evidence that the MIF2 gene of Saccharomyces cerevisiae encodes a centromere protein with homology to the mammalian centromere protein CENP-C. Mol Biol Cell 1995, 6(7):793-807.

38. Dieckhoff P, Bolte M, Sancak Y, Braus GH, Irniger S: Smt3/SUMO and Ubc9 are required for efficient APC/C-mediated proteolysis in budding yeast. Mol Microbiol 2004, 5 I (5): I 375- I 387.

39. Johnson ES, Schwienhorst I, Dohmen RJ, Blobel G: The ubiquitinlike protein $\mathrm{Smt3p}$ is activated for conjugation to other proteins by an AosIp/Uba2p heterodimer. Embo I 1997, I 6( I 8):5509-5519. 
40. Schwienhorst I, Johnson ES, Dohmen RJ: SUMO conjugation and deconjugation. Mol Gen Genet 2000, 263(5):77I-786.

4I. Tanaka K, Nishide J, Okazaki K, Kato H, Niwa O, Nakagawa T, Matsuda $\mathrm{H}$, Kawamukai M, Murakami Y: Characterization of a fission yeast SUMO-I homologue, pmt3p, required for multiple nuclear events, including the control of telomere length and chromosome segregation. Mol Cell Biol I999, I9( I 2):8660-8672.

42. Shayeghi M, Doe CL, Tavassoli M, Watts FZ: Characterisation of Schizosaccharomyces pombe rad3I, a UBA-related gene required for DNA damage tolerance. Nucleic Acids Res 1997, 25(6): I 162-1169.

43. al-Khodairy F, Enoch T, Hagan IM, Carr AM: The Schizosaccharomyces pombe hus 5 gene encodes a ubiquitin conjugating enzyme required for normal mitosis. J Cell Sci 1995, 108 ( Pt 2):475-486.

44. Nacerddine K, Lehembre F, Bhaumik M, Artus J, Cohen-Tannoudji M, Babinet C, Pandolfi PP, Dejean A: The SUMO pathway is essential for nuclear integrity and chromosome segregation in mice. Dev Cell 2005, 9(6):769-779.

45. Chen XL, Reindle A, Johnson ES: Misregulation of $\mathbf{2}$ microm circle copy number in a SUMO pathway mutant. Mol Cell Bio 2005, 25(I0):43II-4320.

46. Azuma Y, Arnaoutov A, Anan T, Dasso M: PIASy mediates SUMO-2 conjugation of Topoisomerase-II on mitotic chromosomes. Embo J 2005, 24(I 2):2 I72-2/82.

47. Diaz-Martinez LA, Gimenez-Abian JF, Azuma Y, Guacci V, GimenezMartin G, Lanier LM, Clarke DJ: PIASgamma is required for faithful chromosome segregation in human cells. PLOS ONE 2006, I:e53.

48. Roth W, Sustmann C, Kieslinger M, Gilmozzi A, Irmer D, Kremmer E, Turck C, Grosschedl R: PIASy-deficient mice display modest defects in IFN and Wnt signaling. J Immunol 2004 I73(10):6189-6199.

49. Arnaoutov A, Azuma Y, Ribbeck K, Joseph J, Boyarchuk Y, Karpova $\mathrm{T}$, McNally J, Dasso M: Crm I is a mitotic effector of Ran-GTP in somatic cells. Nat Cell Biol 2005, 7(6):626-632.

50. Joseph J, Liu ST, Jablonski SA, Yen TJ, Dasso M: The RanGAPIRanBP2 complex is essential for microtubule-kinetochore interactions in vivo. Curr Biol 2004, 14(7):61 I-617.

5I. Rundle NT, Nelson J, Flory MR, Joseph J, Th'ng J, Aebersold R, Dasso $M$, Andersen RJ, Roberge $M$ : An ent-kaurene that inhibits mitotic chromosome movement and binds the kinetochore protein ran-binding protein 2. ACS Chem Biol 2006, I(7):443-450.

52. Salina $D$, Enarson $P$, Rattner JB, Burke $B$ : Nup358 integrates nuclear envelope breakdown with kinetochore assembly. Cell Biol 2003, 162(6):991-100I.

53. Dobson MJ, Pickett AJ, Velmurugan S, Pinder JB, Barrett LA, Jayaram $M$, Chew JS: The 2 microm plasmid causes cell death in Saccharomyces cerevisiae with a mutation in UlpI protease. Mol Cell Biol 2005, 25(I 0):4299-43IO.

54. Li SJ, Hochstrasser M: The yeast ULP2 (SMT4) gene encodes a novel protease specific for the ubiquitin-like Smt3 protein. Mol Cell Biol 2000, 20(7):2367-2377.

55. Bachant J, Alcasabas A, Blat Y, Kleckner N, Elledge S): The SUMOI isopeptidase Smt4 is linked to centromeric cohesion through SUMO-I modification of DNA topoisomerase II. Mol Cell 2002, 9(6): I I69-I I82.

56. Taylor DL, Ho JC, Oliver A, Watts FZ: Cell-cycle-dependent localisation of UlpI, a Schizosaccharomyces pombe Pmt3 (SUMO)-specific protease. J Cell Sci 2002, I I 5(Pt 6): I I I3- I I 22.

57. Losada A, Hirano T: Dynamic molecular linkers of the genome: the first decade of SMC proteins. Genes Dev 2005 I 9(II): I 269-I 287.

58. Stead K, Aguilar C, Hartman T, Drexel M, Meluh P, Guacci V: Pds5p regulates the maintenance of sister chromatid cohesion and is sumoylated to promote the dissolution of cohesion. J Cell Biol 2003, 163(4):729-74I.

59. D'Amours D, Stegmeier F, Amon A: $\mathrm{Cdcl} 4$ and condensin control the dissolution of cohesin-independent chromosome linkages at repeated DNA. Cell 2004, I I 7(4):455-469.

60. Azuma Y, Arnaoutov A, Dasso M: SUMO-2/3 regulates topoisomerase II in mitosis. J Cell Biol 2003, I63(3):477-487.

61. Cleveland DW, Mao Y, Sullivan KF: Centromeres and kinetochores: from epigenetics to mitotic checkpoint signaling. Cell 2003, I I 2(4):407-42I.
62. McDonald KL, O'Toole ET, Mastronarde DN, Mclntosh JR: Kinetochore microtubules in PTK cells. J Cell Biol 1992, I I 8(2):369-383.

63. Montpetit B, Hazbun TR, Fields S, Hieter P: Sumoylation of the budding yeast kinetochore protein $\mathrm{Ndcl} 0$ is required for Ndc 10 spindle localization and regulation of anaphase spindle elongation. J Cell Biol 2006, 174(5):653-663.

64. Bouck D, Bloom K: The role of centromere-binding factor 3 (CBF3) in spindle stability, cytokinesis, and kinetochore attachment. Biochem Cell Biol 2005, 83(6):696-702.

65. Sandall S, Severin F, McLeod IX, Yates JR 3rd, Oegema K, Hyman A, Desai A: A Birl-Slil5 complex connects centromeres to microtubules and is required to sense kinetochore tension. Cell 2006, I27(6): ||79-1|9|.

66. Ruchaud S, Carmena M, Earnshaw WC: Chromosomal passengers: conducting cell division. Nat Rev Mol Cell Biol 2007, 8(10):798-8I2

67. Sun $\mathrm{H}$, Leverson JD, Hunter $\mathrm{T}$ : Conserved function of RNF4 family proteins in eukaryotes: targeting a ubiquitin ligase to SUMOylated proteins. Embo / 2007, 26 ( I 8):4 |02-4I I 2 .

68. Uzunova K, Gottsche K, Miteva M, Weisshaar SR, Glanemann C, Schnellhardt M, Niessen M, Scheel H, Hofmann K, Johnson ES, Praefcke G], Dohmen RJ: Ubiquitin-dependent proteolytic control of SUMO conjugates. J Biol Chem 2007, 282(47):34167-34I75.

69. Xie Y, Kerscher O, Kroetz MB, McConchie HF, Sung P, Hochstrasser $M$ : The yeast Hex3.S1x8 heterodimer is a ubiquitin ligase stimulated by substrate sumoylation. I Biol Chem 2007, 282(47):34|76-34|84.

70. Politi V, Perini G, Trazzi S, Pliss A, Raska I, Earnshaw WC, Della Valle G: CENP-C binds the alpha-satellite DNA in vivo at specific centromere domains. J Cell Sci 2002, I I 5(Pt I I):23 17-2327.

7I. Kwon MS, Hori T, Okada M, Fukagawa T: CENP-C is involved in chromosome segregation, mitotic checkpoint function, and kinetochore assembly. Mol Biol Cell 2007, 18(6):2155-2168.

72. Fukagawa $T$, Regnier $V$, lkemura $T$ : Creation and characterization of temperature-sensitive CENP-C mutants in vertebrate cells. Nucleic Acids Res 200I, 29( I8):3796-3803.

73. Chung TL, Hsiao HH, Yeh YY, Shia HL, Chen YL, Liang PH, Wang AH, Khoo KH, Shoei-Lung Li S: In vitro modification of human centromere protein CENP-C fragments by small ubiquitin-like modifier (SUMO) protein: definitive identification of the modification sites by tandem mass spectrometry analysis of the isopeptides. J Biol Chem 2004, 279(38):39653-39662.

74. Kline-Smith SL, Sandall S, Desai A: Kinetochore-spindle microtubule interactions during mitosis. Curr Opin Cell Biol 2005, I7(I):35-46.

75. Maiato H, DeLuca J, Salmon ED, Earnshaw WC: The dynamic kinetochore-microtubule interface. I Cell Sci 2004, I I 7(Pt 23):546I-5477.

76. Cooke CA, Schaar B, Yen TJ, Earnshaw WC: Localization of CENP-E in the fibrous corona and outer plate of mammalian kinetochores from prometaphase through anaphase. Chromosoma 1997, 106(7):446-455.

77. Yen TJ, Compton DA, Wise D, Zinkowski RP, Brinkley BR, Earnshaw WC, Cleveland DW: CENP-E, a novel human centromereassociated protein required for progression from metaphase to anaphase. Embo J 199I, 10(5): I245-1254.

78. Kapoor TM, Lampson MA, Hergert P, Cameron L, Cimini D, Salmon ED, McEwen BF, Khodjakov A: Chromosomes can congress to the metaphase plate before biorientation. Science 2006, 3 II (5759):388-39I.

79. McEwen BF, Chan GK, Zubrowski B, Savoian MS, Sauer MT, Yen TJ: CENP-E is essential for reliable bioriented spindle attachment, but chromosome alignment can be achieved via redundant mechanisms in mammalian cells. Mol Biol Cell 200I, I 2(9):2776-2789.

80. Putkey FR, Cramer T, Morphew MK, Silk AD, Johnson RS, Mclntosh $J R$, Cleveland DW: Unstable kinetochore-microtubule capture and chromosomal instability following deletion of CENP-E. Dev Cell 2002, 3(3):35I-365.

8I. Zhang XD, Goeres J, Zhang H, Yen TJ, Porter ACG, Matunis MJ: SUMO-2/3 Modification and Binding Regulates the Association of CENP-E with Kinetochores and Progression through Mitosis. submitted 2007. 
82. Matunis MJ, Wu J, Blobel G: SUMO-I modification and its role in targeting the Ran GTPase-activating protein, RanGAPI, to the nuclear pore complex. J Cell Biol 1998, I 40(3):499-509.

83. Saitoh H, Sparrow DB, Shiomi T, Pu RT, Nishimoto T, Mohun T] Dasso M: Ubc9p and the conjugation of SUMO-I to RanGAP I and RanBP2. Curr Biol 1998, 8(2): | $2|-| 24$.

84. Arnaoutov A, Dasso M: Ran-GTP regulates kinetochore attachment in somatic cells. Cell Cycle 2005, 4(9): I I6I-I I65.

85. Jeong SY, Rose A, Joseph J, Dasso M, Meier I: Plant-specific mitotic targeting of RanGAP requires a functional WPP domain. Plant J 2005, 42(2):270-282.

86. Versele $M$, Thorner J: Some assembly required: yeast septins provide the instruction manual. Trends Cell Biol 2005, I 5(8):4|4-424.

87. Johnson ES, Blobel G: Cell cycle-regulated attachment of the ubiquitin-related protein SUMO to the yeast septins. J Cell Biol 1999, I47(5):981-994.

88. Keaton MA, Lew DJ: Eavesdropping on the cytoskeleton: progress and controversy in the yeast morphogenesis checkpoint. Curr Opin Microbiol 2006, 9(6):540-546.

89. Makhnevych T, Ptak C, Lusk CP, Aitchison JD, Wozniak RW: The role of karyopherins in the regulated sumoylation of septins. J Cell Biol 2007, I 77(I):39-49.

90. Shih HP, Hales KG, Pringle JR, Peifer M: Identification of septininteracting proteins and characterization of the Smt3 SUMO-conjugation system in Drosophila. J Cell Sci 2002 I I 5(Pt 6): | 259-|27|.

9I. Cheng TS, Chang LK, Howng SL, Lu PJ, Lee CI, Hong YR: SUMO-I modification of centrosomal protein hNinein promotes $\mathrm{hNi}$ nein nuclear localization. Life Sci 2006, 78( I 0): I I I4- I I 20.

92. Sillibourne JE, Delaval B, Redick S, Sinha M, Doxsey SJ: Chromatin remodeling proteins interact with pericentrin to regulate centrosome integrity. Mol Biol Cell 2007, I 8(9):3667-3680.

93. Minty A, Dumont X, Kaghad M, Caput D: Covalent modification of p73alpha by SUMO-I. Two-hybrid screening with p73 identifies novel SUMO-I-interacting proteins and a SUMO-I interaction motif. I Biol Chem 2000, 275(46):363 I 6-36323.

94. Panse VG, Kuster B, Gerstberger T, Hurt E: Unconventional tethering of Ulpl to the transport channel of the nuclear pore complex by karyopherins. Nat Cell Biol 2003, 5(I):2I-27.

95. Nathan D, Ingvarsdottir K, Sterner DE, Bylebyl GR, Dokmanovic M, Dorsey JA, Whelan KA, Krsmanovic M, Lane WS, Meluh PB, Johnson $E S$, Berger SL: Histone sumoylation is a negative regulator in Saccharomyces cerevisiae and shows dynamic interplay with positive-acting histone modifications. Genes Dev 2006 , 20(8):966-976.

\section{Publish with Biomed Central and every scientist can read your work free of charge}

"BioMed Central will be the most significant development for disseminating the results of biomedical research in our lifetime. "

Sir Paul Nurse, Cancer Research UK

Your research papers will be:

- available free of charge to the entire biomedical community

- peer reviewed and published immediately upon acceptance

- cited in PubMed and archived on PubMed Central

- yours - you keep the copyright
BioMedcentral 